\title{
Sound radiation from a turbulent boundary layer
}

\author{
Zhiwei $\mathrm{Hu}^{\text {a) }}$ \\ Aerodynamics and Flight Mechanics Research Group, School of Engineering Sciences, \\ University of Southampton, Southampton SO17 1BJ, United Kingdom \\ Christopher L. Morfey \\ Fluid Dynamics and Acoustics Group, ISVR, University of Southampton, \\ Southampton SO17 1BJ, United Kingdom \\ Neil D. Sandham \\ Aerodynamics and Flight Mechanics Research Group, School of Engineering Sciences, \\ University of Southampton, Southampton SO17 1BJ, United Kingdom
}

(Received 11 January 2006; accepted 21 July 2006; published online 7 September 2006)

\begin{abstract}
Sound radiation due to fluctuating viscous wall shear stresses in a plane turbulent boundary layer is investigated by a two-stage procedure using direct numerical simulation (DNS) databases for incompressible turbulent Poiseuille flow in a plane channel, at Reynolds numbers up to $\operatorname{Re}_{\tau}=1440$. The power spectral density of radiated pressure and spectra of sound power per unit wall area are calculated in the low Mach number limit by substituting source terms obtained from DNS into a Ffowcs Williams-Hawkings wave equation and using a half-space Green function. The same DNS data are used to predict the spectrum of turbulent boundary layer noise measured in a diffuser downstream of a fully developed channel flow [Greshilov and Mironov, Sov. Phys. Acoust. 29, 275 (1983)]. The measured spectrum is $\sim 15 \mathrm{~dB}$ higher at low frequencies, but converges with the prediction at high frequencies. (C) 2006 American Institute of Physics. [DOI: 10.1063/1.2337733]
\end{abstract}

Sound radiated by a turbulent boundary layer differs from that radiated by free turbulence owing to the existence of the solid wall, which introduces a dipole source layer of wall shear stresses; ${ }^{1-3}$ the acoustic conversion efficiency (defined as the ratio of sound power radiated to power dissipated through drag, per unit wall area) is predicted to vary as $\mathrm{M}^{3}$ for the boundary layer as compared with $\mathrm{M}^{5}$ for free turbulence, ${ }^{4,5}$ where $M$ is the free-stream Mach number. However, measuring turbulent boundary layer noise, especially at low Mach numbers, is very difficult because of its small amplitude, the inevitable existence of background noise, and disturbances caused by transducers and the edges or supports of the model. Despite carefully controlled experiments, ${ }^{6-8}$ a large scatter exists in the measured data.

In this Brief Communication, sound radiation from a flat-plate turbulent boundary layer is predicted by a hybrid method (in the terminology of Ref. 9), using direct numerical simulation (DNS) databases established by the current authors ${ }^{10}$ for incompressible Poiseuille flow in a plane channel. Results have been obtained in the limit $\mathrm{M} \ll 1$, by focusing on the contribution of lowest order in M. As sketched in Fig. 1, we use half of the channel as a model for a flat-plate boundary layer and assume: (i) the streamwise development of the turbulent boundary layer is slow on the characteristic scale of turbulence correlation lengths; (ii) turbulence statistics are homogeneous in the spanwise direction; and (iii) the relevant unsteady shear-stress statistics at the wall are not sensitive to streamwise pressure gradient, as explained below.

The mean pressure gradient for Poiseuille flow at all

${ }^{a)}$ Electronic mail: z.hu@soton.ac.uk
Reynolds numbers has the same nondimensional value $d\langle P\rangle / d x_{1}=-1$. Here we use nondimensional variables scaled by the friction velocity $u_{\tau}^{*}=\sqrt{\tau_{w}^{*} / \rho_{0}^{*}}$, the density of the undisturbed fluid outside the boundary layer $\rho_{0}^{*}$, and the channel half-width $h^{*}$; the asterisk denotes dimensional quantities. Over the current Reynolds number range $\operatorname{Re}_{\tau}=u_{\tau}^{*} h^{*} / \nu^{*}$ $=360 \sim 1440$ (corresponding to $\operatorname{Re}_{\theta}=U_{\max }^{*} \theta^{*} / \nu^{*}=626-2936$; $U_{\max }^{*}$ is the channel centerline velocity, and $\theta^{*}$ is the momentum thickness of the boundary layer), Clauser's parameter $\left(\beta=\delta_{1}^{*}\left\langle d P^{*} / d x_{1}^{*}\right\rangle / \tau_{w}^{*}\right.$, where $\delta_{1}^{*}$ is the displacement thickness $)$ changes only from -0.087 for $\mathrm{Re}_{\tau}=360$ to -0.086 for $\mathrm{Re}_{\tau}$ $=1440$. Thus, all our simulations operate under almost the same weak favorable pressure gradient, and we assume that fluctuating wall-stress statistics for the channel can be used to predict sound radiation from a zero pressure gradient turbulent boundary layer at the same $\operatorname{Re}_{\theta}$.

With respect to assumption (i) above, based on the 1/7-power law for flat-plate turbulent boundary layer, the fractional growth in boundary layer thickness over distance $l_{x}, l_{x} d(\ln \delta) / d x$, is estimated to be 0.064 for $\mathrm{Re}_{\tau}=360$ and 0.0125 for $\operatorname{Re}_{\tau}=1440$, decreasing with Reynolds number. Here, $l_{x}$ is the streamwise separation at which the modulus of the complex coherence function for wall shear stress drops to $1 / e$ of its peak value. This quantity characterizes the correlation length of wall shear stresses, and decreases with frequency as $f^{-0.7}$ (results not shown here due to limited space); a representative value of $l_{x}^{+}=l_{x}^{*} u_{\tau}^{*} / \nu^{*}=1000$ at low frequencies $\left(f^{+} \simeq 3 \times 10^{-3}\right)$ is used in the above estimation.

To describe sound radiation in the limit $\mathrm{M} \ll 1$, we adopt the formulation of Ref. 2. Referring to Fig. 1, a generalized wave variable $p H\left(x_{3}\right)$ is introduced; $p=\left(P^{*}-P_{0}^{*}\right) / \tau_{w}^{*}$ is the nondimensional acoustic pressure, and $H\left(x_{3}\right)$ is a Heaviside 

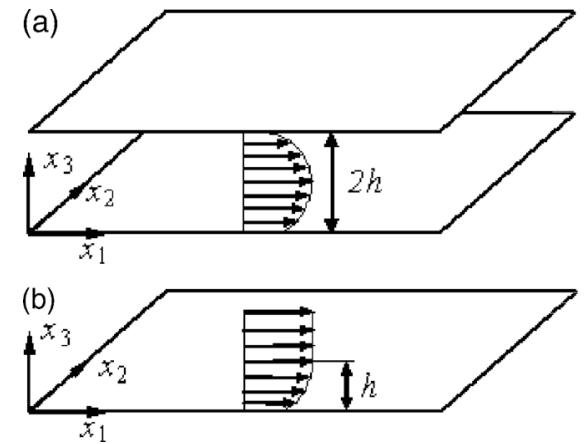

FIG. 1. Sketch for turbulent channel flow (a) and boundary layer (b) models.

function equal to 1 in $x_{3} \geq 0$, and 0 elsewhere. The following inhomogeneous wave equation can be derived for adiabatic flow in the low Mach number limit, with source terms given to order $\mathrm{M}^{2}$ :

$$
\begin{gathered}
\left(\mathrm{M}_{\tau}^{2} \frac{\partial^{2}}{\partial t^{2}}-\frac{\partial^{2}}{\partial x_{i}^{2}}\right)(p H)=\frac{\partial^{2}\left(T_{i j} H\right)}{\partial x_{i} \partial x_{j}}-\frac{\partial\left(F_{i} H+B_{i} \delta\right)}{\partial x_{i}} \\
+\frac{\partial(Q H)}{\partial t} ; \\
T_{i j} \simeq u_{i} u_{j}-\tau_{i j}, \quad F_{i} \simeq G_{i}, \quad Q \simeq \lambda \mathrm{M}_{\tau}^{2} \Phi ; \\
B_{i}=-\tau_{i j} b_{j}+p b_{i} \quad\left(b_{i}=\text { unit wall normal }\right) .
\end{gathered}
$$

Here, $H\left(x_{3}\right)$ and its derivative $\delta\left(x_{3}\right)$ are abbreviated as $H, \delta$; $\mathrm{M}_{\tau}=u_{\tau}^{*} / c_{0}^{*}$, and $c_{0}^{*}$ is the free-stream sound speed. On the right of (1), $\left(T_{i j}, F_{i}, Q\right)$ are volume quadrupole, dipole, and monopole sources, respectively, and $B_{i}$ is a wall dipole layer. In (2) and (3), $u_{i}, \tau_{i j}$, and $G_{i}(i, j=1$ to 3$)$ denote fluid velocity, viscous stress, and body force per unit volume, respectively; $\Phi$ is the viscous dissipation function, and $\lambda=(\rho / T)$ $\times(\partial T / \partial \rho)_{s}$ is the Grüneisen parameter, ${ }^{11}$ equal to $\gamma-1$ for a perfect gas with specific-heat ratio $\gamma$.

The radiated pressure at position $\boldsymbol{x}$ is given in the frequency domain by

$$
\begin{aligned}
p(\boldsymbol{x}, f)= & \int_{V}\left\{T_{i j} \frac{\partial^{2} g}{\partial y_{i} y_{j}}+\left(F_{i}+B_{i} \delta\right) \frac{\partial g}{\partial y_{i}}-\mathrm{i} 2 \pi f Q g\right\} d^{3} \boldsymbol{y} \\
& \left(x_{3} \geq 0\right),
\end{aligned}
$$

where $f$ is frequency and $g(\boldsymbol{x} \mid \boldsymbol{y})$ is the half-space Green function with Neumann boundary condition at the wall. ${ }^{12}$ The integration is restricted to the boundary layer of thickness $h$, as no acoustic source exists outside. As $\mathrm{M} \rightarrow 0$, the nondimensional acoustic wavenumber $k_{0}=2 \pi f \mathrm{M}_{\tau}$ tends to zero. For $r=|\boldsymbol{x}-\boldsymbol{y}| \gg y_{3}$, the far-field approximation $r \simeq r_{0}-e_{3} y_{3}$ gives

$$
\begin{aligned}
& g(\boldsymbol{x} \mid \boldsymbol{y}) \simeq g\left(\boldsymbol{x} \mid \boldsymbol{y}_{0}\right)=\frac{e^{\mathrm{i} k_{0} r_{0}}}{2 \pi r_{0}}, \\
& \frac{\partial g(\boldsymbol{x} \mid \boldsymbol{y})}{\partial y_{\alpha}} \simeq-\mathrm{i} k_{0} e_{\alpha} g\left(\boldsymbol{x} \mid \boldsymbol{y}_{0}\right), \quad(\alpha=1,2) ;
\end{aligned}
$$

$$
\frac{\partial g(\boldsymbol{x} \mid \boldsymbol{y})}{\partial y_{3}} \simeq-k_{0}^{2} y_{3} e_{3}^{2} g\left(\boldsymbol{x} \mid \boldsymbol{y}_{0}\right) .
$$

Here, $\boldsymbol{y}_{0}=\left(y_{1}, y_{2}, 0\right)$ is the projection of $\boldsymbol{y}$ on the wall; $r_{0}=\left|\boldsymbol{x}-\boldsymbol{y}_{0}\right|$, and $\boldsymbol{e}=\left(\boldsymbol{x}-\boldsymbol{y}_{0}\right) / r_{0}$ is the unit radiation vector.

The asymptotic $k_{0}^{2}$ dependence in Eq. (7), compared to the $k_{0}$ dependence for derivatives parallel to the wall in Eq. (6), introduces an $\mathrm{M}^{2}$ factor in the wall-normal derivative of the Green function that has an important impact on the radiated pressure. When Eqs. (5)-(7) are substituted in the integral of Eq. (4), it follows that components $T_{i j}$ with one of $i$ or $j=3$ do not contribute to the far-field pressure to order $\mathrm{M}^{2}$. The normal dipole layer $B_{3} \delta$ also drops out because $\partial g / \partial y_{3}$ $=0$ at $y_{3}=0$. The volume dipole source $F_{i}=G_{i}$ as a steady force field equivalent to the mean pressure gradient, has no contribution either. The far-field radiated pressure can be estimated to order $\mathrm{M}^{2}$ as

$$
\begin{aligned}
p(\boldsymbol{x}, f) \simeq & \int_{V}\left\{\left(\mathrm{i} k_{0}\right)^{2}\left(e_{\alpha} e_{\beta} T_{\alpha \beta}+e_{3}^{2} T_{33}\right)-\mathrm{i} k_{0} e_{\alpha} B_{\alpha} \delta\right. \\
& -\mathrm{i} 2 \pi f Q\} g\left(\boldsymbol{x} \mid \boldsymbol{y}_{0}\right) d^{3} \boldsymbol{y}, \quad(\alpha, \beta=1,2), \\
\simeq & \iint \Gamma\left(y_{1}, y_{2}, f\right) g\left(\boldsymbol{x} \mid \boldsymbol{y}_{0}\right) d y_{1} d y_{2},
\end{aligned}
$$

where repeated $\alpha, \beta$ subscripts imply summation over $(\alpha, \beta)=1,2$, and $p, T_{\alpha \beta}, B_{\alpha}$, and $Q$ are Fourier transforms of the corresponding time-domain quantities. In Eq. (8), $\Gamma\left(y_{1}, y_{2}, f\right)$ is an equivalent two-dimensional source distribution in the plane $y_{3}=0$, obtained from Eqs. (2) and (3) by integration through the boundary layer:

$$
\Gamma\left(y_{1}, y_{2}, f\right)=\left(\mathrm{i} k_{0}\right)^{2}\left(e_{\alpha} e_{\beta} t_{\alpha \beta}+e_{3}^{2} t_{33}\right)-\mathrm{i} k_{0} e_{\alpha} \sigma_{\alpha}-\mathrm{i} 2 \pi f q .
$$

The source terms $t_{i j}, \sigma_{\alpha}$ and $q$ in Eq. (9) are given to $O\left(\mathrm{M}^{2}\right)$ accuracy by

$$
\begin{aligned}
& t_{i j} \simeq \int_{0}^{h}\left(u_{i} u_{j}-\tau_{i j}\right) d y_{3}, \quad \sigma_{\alpha}=-\left.\tau_{\alpha 3}\right|_{y_{3}=0} ; \\
& q \simeq \lambda \mathrm{M}_{\tau}^{2} \Psi=\lambda \mathrm{M}_{\tau}^{2} \int_{0}^{h} \Phi d y_{3} .
\end{aligned}
$$

Statistical properties of the radiation are obtained by sampling the boundary layer in $\left(y_{1}, y_{2}, t\right)$ space: $0 \leq y_{1} \leq L_{1}$, $0 \leq y_{2} \leq L_{2}, 0 \leq t \leq T$. Later, $L_{1}, L_{2}, T$ will be identified with the computational box size and runtime. The two-sided power spectral density of radiated pressure at $\boldsymbol{x}$, normalized to unit wall area, is given by the following mathematical expectation:

$$
s_{p}(\boldsymbol{x}, f)=\mathrm{E}\left\{\lim _{T, L_{1}, L_{2} \rightarrow \infty} \frac{1}{T L_{1} L_{2}}|p(\boldsymbol{x}, f)|^{2}\right\} .
$$

We assume $|\boldsymbol{x}|=R \gg\left(L_{1}, L_{2}\right) \gg h$, so that $\boldsymbol{e} \simeq \boldsymbol{x} / R$. Also in Eq. (5), $r_{0}$ can be replaced by $R$ in the denominator and by $R-e_{\alpha} y_{\alpha}(\alpha=1,2)$ in the phase term; Eq. (8) then gives 


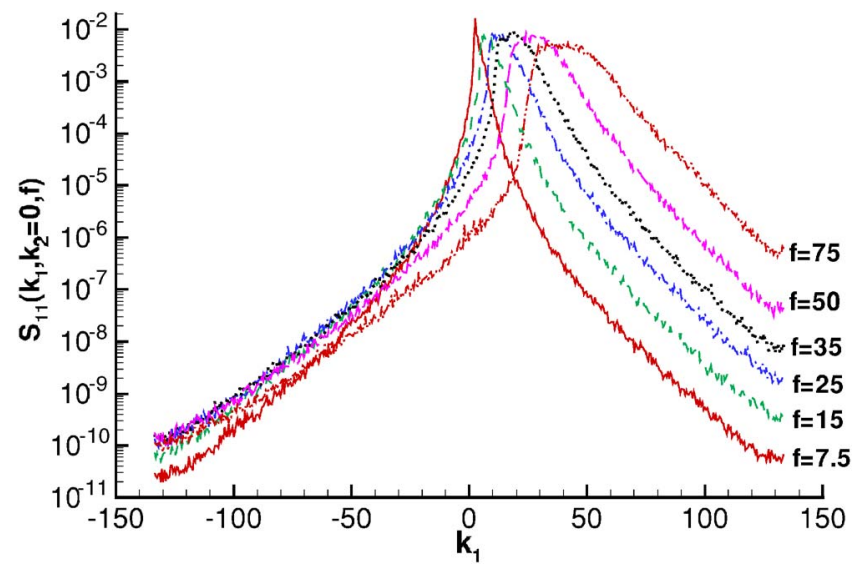

FIG. 2. (Color online) Wavenumber-frequency spectrum $S_{11}$ of the streamwise wall shear stress dipole source at $\operatorname{Re}_{\tau}=720$. Note that all spectra in $\left(k_{1}, k_{2}, f\right)$ are two-sided.

$$
\begin{aligned}
p(\boldsymbol{x}, f) & =\frac{e^{\mathrm{i} k_{0} R}}{2 \pi R} \iint \Gamma\left(y_{1}, y_{2}, f\right) e^{-\mathrm{i} k_{0}\left(e_{1} y_{1}+e_{2} y_{2}\right)} d y_{1} d y_{2} \\
& =\frac{e^{\mathrm{i} k_{0} R}}{2 \pi R} \Gamma\left(e_{1} k_{0}, e_{2} k_{0}, f\right),
\end{aligned}
$$

where $\Gamma\left(k_{1}, k_{2}, f\right)$ is the two-dimensional spatial Fourier transform of $\Gamma\left(y_{1}, y_{2}, f\right)$. The $t_{i j}$ and $q$ contributions to $\Gamma$ in Eq. (9) are both $O\left(\mathrm{M}_{\tau}^{2}\right)$, while the $\sigma_{\alpha}$ contribution is $O\left(\mathrm{M}_{\tau}\right)$; therefore, the far-field radiation is dominated by the shearstress dipole contribution in the low-M limit. We focus on this contribution for the remainder of the Brief Comunication. The power spectral density of radiated pressure per unit wall area follows from Eq. (12) as

$$
\begin{aligned}
s_{p}(\boldsymbol{x}, f) & =\frac{1}{(2 \pi R)^{2}} \mathrm{E}\left\{\lim _{T, L_{1}, L_{2} \rightarrow \infty} \frac{1}{T L_{1} L_{2}}\left|\Gamma\left(e_{1} k_{0}, e_{2} k_{0}, f\right)\right|^{2}\right\} \\
& =R^{-2} S_{\Gamma}\left(e_{1} k_{0}, e_{2} k_{0}, f\right) .
\end{aligned}
$$

The quantity $S_{\Gamma}\left(e_{1} k_{0}, e_{2} k_{0}, f\right)$ introduced above is the wavenumber-frequency power spectral density of the equivalent two-dimensional source $\Gamma$. The radiation wavenumber $\left(e_{1} k_{0}, e_{2} k_{0}\right)$ is now replaced by $(0,0)$ on the assumptionjustified in Ref. 13-that the wall stress $\sigma_{\alpha}$ in $\Gamma\left(y_{1}, y_{2}, f\right)$ has a nonzero wavenumber spectral density in the zerowavenumber limit. This term then dominates $S_{\Gamma}$ as $\mathrm{M} \rightarrow 0$, giving

$$
S_{\Gamma} \simeq \mathrm{M}_{\tau}^{2}\left(e_{1}^{2} S_{11}+e_{2}^{2} S_{22}\right)+O\left(\mathrm{M}^{4}\right),
$$

where $S_{\alpha \beta}=(2 \pi f)^{2} S_{\sigma_{\alpha} \sigma_{\beta}}$ are the wavenumber-frequency auto- and cross-spectra, respectively, of $\dot{\sigma}_{\alpha}, \dot{\sigma}_{\beta}(\alpha, \beta=1,2$, dot denotes time derivative), evaluated at zero wavenumber. No $S_{12}$ term appears in Eq. (14), because statistical symmetry in the spanwise direction makes $S_{12}$ vanish at $k_{2}=0$. Estimates of the $S_{11}$ and $S_{22}$ zero-wavenumber spectra have been calculated from DNS databases established by the current authors ${ }^{10}$ for incompressible turbulent Poiseuille flow at Reynolds numbers up to $\operatorname{Re}_{\tau}=1440$. Time records for source terms in Eq. (10) are stored in wave space $\left(k_{1}, k_{2}\right)$, once the flow has reached a fully developed state. To reduce the statistical variance of the spectral estimates, the time record for

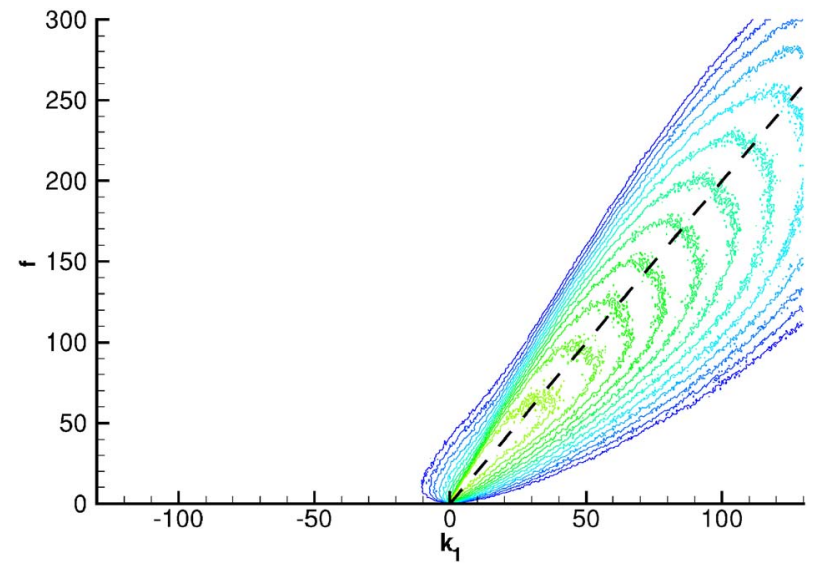

FIG. 3. (Color online) Contours of the $S_{11}$ wavenumber-frequency spectrum at $k_{2}=0$, calculated for the streamwise dipole source at $\operatorname{Re}_{\tau}=720$. Seventeen exponentially distributed contours are plotted between $10^{-1}$ and $10^{-5}$. Slope of the dashed line equals $U_{\text {con }} / 2 \pi$. Similar convection features apply to $S_{22}$.

each source term is divided into several (typically seven) time segments with 50\% overlap, and spectra calculated from each time segment are averaged. More details can be found in Refs. 10 and 13. To investigate the validity of using channel-flow DNS data to represent wall shear-stress statistics for a boundary layer, the cross-correlation coefficient of stresses on opposite walls has been calculated. The crosscorrelation coefficient is virtually zero (less than 0.015 for all cases). For the spatial mean (wall-averaged) shear stresses, no converged correlation coefficients have been obtained owing to the limited run time available. The calculated values oscillate around zero as the number of samples is increased.

As indicated by Eq. (14), the spectrum of the wall shear stress at zero wavenumber is of particular interest for hydrodynamic sound radiation. To improve our low-wavenumber estimates of $S_{11}$ and $S_{22}$, a least-squares fit in the form $a k_{2}^{2}$ $+b$ was performed at each $\left(k_{1}, f\right)$ in the vicinity of zero $k_{2}$, based on the fact that the flow is homogeneous in the spanwise direction, and thus its spectra are symmetric about $k_{2}=0$ (see examples of $k_{2}$ spectra in Ref. 2). The number of points involved varies between 10 and 50, increasing with frequency.

Figure 2 shows a typical set of wavenumber-frequency spectra. The streamwise dipole spectrum $S_{11}$ is plotted against $k_{1}$ with $k_{2}=0$, at six frequencies for $\operatorname{Re}_{\tau}=720$. The peaks seen at $k_{1}>0$ correspond to the convective character of the wall-stress field. A contour plot of $S_{11}\left(k_{1}, 0, f\right)$ (Fig. 3) shows all peaks lying in a preferred direction, whose slope corresponds to the convection velocity of wall shear stress.

Frequency spectra for the streamwise and spanwise dipole source terms at zero wavenumber are shown in Fig. 4, for $\operatorname{Re}_{\tau}=360,720$, and 1440. Data are plotted in normalized form, $f^{*} S_{\alpha \alpha}^{*}\left(0,0, f^{*}\right) /\left(u_{\tau}^{*} \tau_{w}^{*}\right)^{2}$, against $f^{+}=f^{*} \nu^{*} / u_{\tau}^{* 2}$. From Eqs. (13) and (14), the $y$ axis represents the (frequencyweighted) power spectral density of radiated pressure per unit wall area in the streamwise (a) and spanwise (b) directions, respectively, normalized as $f^{*} s_{p}^{*} /\left(\tau_{w}^{*} \mathrm{M}_{\tau} / R^{*}\right)^{2}$. Note that the use of viscous scaling collapses the spectra for different $\operatorname{Re}_{\tau}$. Comparison of Figs. 4(a) and 4(b) shows the radiated 

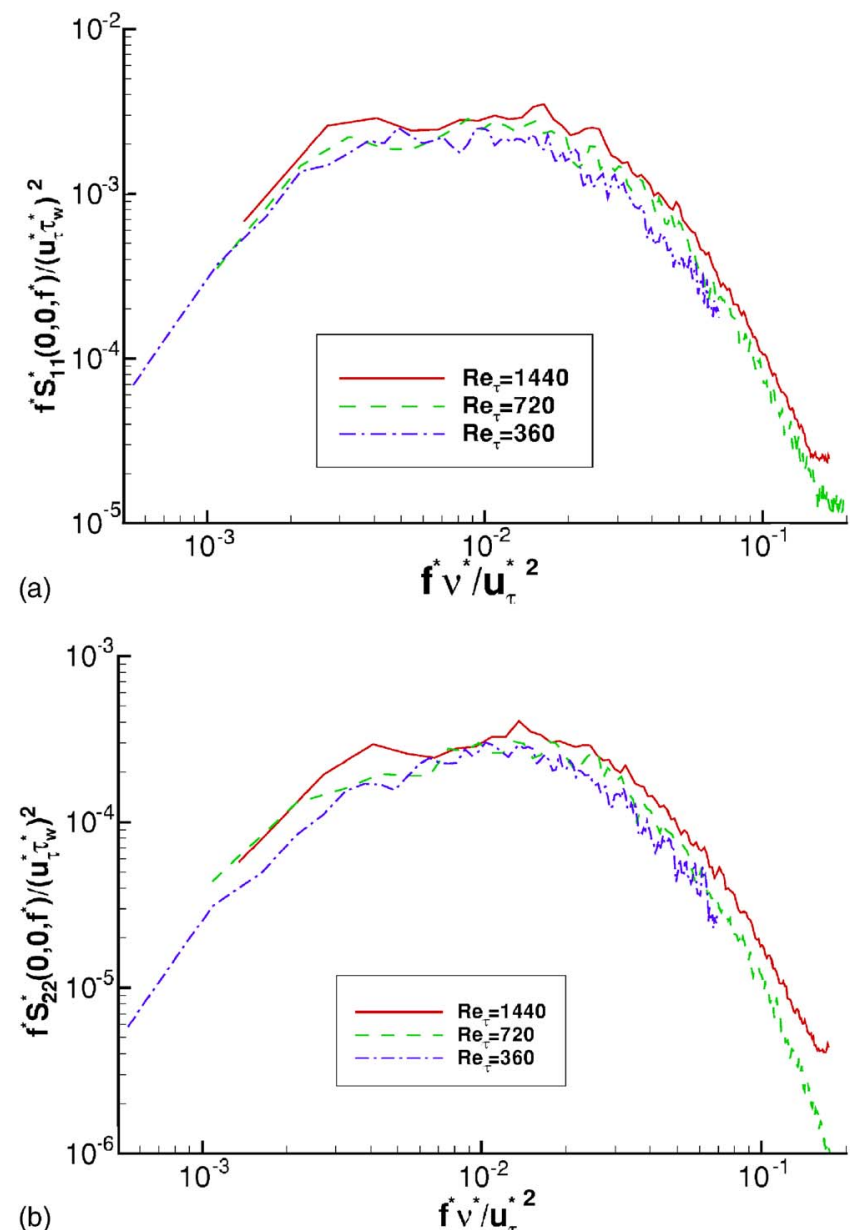

FIG. 4. (Color online) Frequency-weighted wavenumber-frequency spectra of the streamwise (a) and the spanwise (b) wall shear stress dipole sources. According to Eqs. (13) and (14), the ordinate represents the normalized power spectral density of radiated pressure $f^{*} s_{p}^{*} /\left(\tau_{w}^{*} M_{\tau} / R^{*}\right)^{2}$, per unit area of wall.

intensity to be more than $10 \mathrm{~dB}$ higher in the streamwise than in the spanwise direction.

The sound intensity spectrum per unit wall area follows from Eqs. (13) and (14) as $\mathrm{M}_{\tau} s_{p}$. Integrating the acoustic intensity over a hemisphere yields the spectrum of radiated sound power emitted from a turbulent boundary layer per unit wall area, $w(f)$, given by

$$
w(f)=\left(2 \pi \mathrm{M}_{\tau}^{3} / 3\right)\left(S_{11}+S_{22}\right)+O\left(\mathrm{M}^{5}\right) .
$$

At low Mach numbers, the two surface dipole sources are the main contributors. The (frequency-weighted) spectrum of radiated sound power will therefore collapse under the scaling used in Fig. 4, when plotted as $f^{*} w^{*}\left(f^{*}\right) /\left(\mathbf{M}_{\tau}^{3} u_{\tau}^{*} \tau_{w}^{*}\right)$ against $f^{+}$.

These DNS predictions can be compared with the measurements of Greshilov and Mironov ${ }^{8}$ for the radiated hydrodynamic sound from a water channel made in a downstream diffuser. The transfer function between streamwise wall shear stress fluctuations in the channel and acoustic pressure in the diffuser for the experimental acoustic system was estimated by matching the reported resonance frequencies and $Q$ factors for the first resonance and the one near $1 \mathrm{kHz}$. DNS data for the streamwise dipole source spectrum $S_{11}$

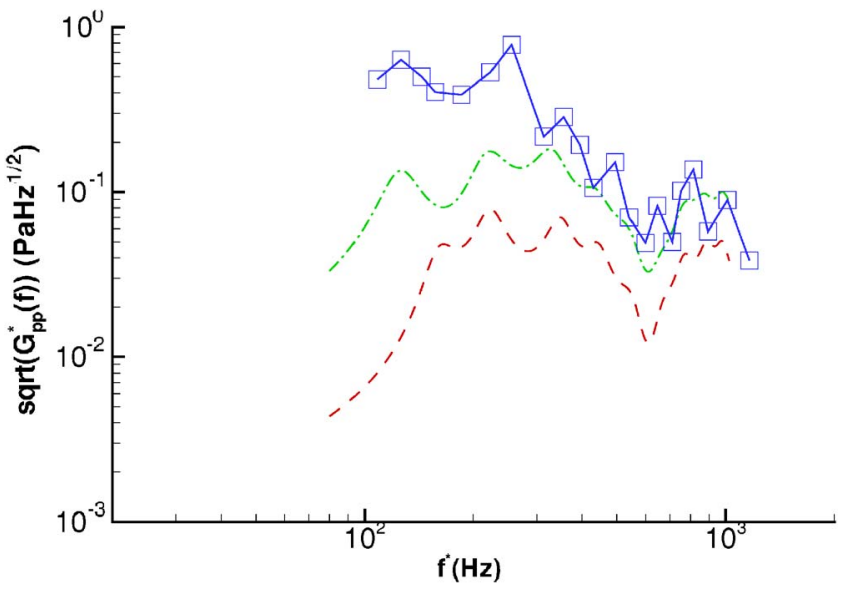

FIG. 5. (Color online) Comparison of the radiated pressure spectrum in the diffuser from DNS predictions with the experiment of Greshilov and Mironov (Ref. 8) (solid line with symbols). Predictions are based on equivalent conical-diffuser angles of $0.3^{\circ}$ (dash-dotted line) and $1.5^{\circ}$ (dashed line), measured between the axis and diffuser wall. $\mathbf{M}_{\infty}=0.008$.

were then extrapolated to the experimental Reynolds number $\left(\operatorname{Re}_{\tau}=4300\right)$ based on the scaling given in Fig. 4. Results are shown in Fig. 5 for the radiated pressure spectrum at the measuring point in the diffuser. Two predicted spectra have been plotted to account for the uncertainty in the diffuser geometry. The reported diffuser angle is $1.5^{\circ}\left(3^{\circ}\right.$ included angle); however, this leads to a frequency for the first resonance that is too high. When the diffuser angle is taken to be $0.3^{\circ}$, the correct resonance frequencies are predicted. The experimental pressure spectra are close to the prediction at high frequencies. The excess noise measured below $300 \mathrm{~Hz}$ may be due to transitional or other extraneous effects in the upstream part of the channel.

${ }^{1}$ M. Wang, S. K. Lele, and P. Moin, "Sound radiation during local laminar breakdown in a low-Mach-number boundary layer," J. Fluid Mech. 319, 197 (1996).

${ }^{2}$ Z. W. Hu, C. L. Morfey, and N. D. Sandham, "Sound radiation in turbulent channel flows," J. Fluid Mech. 475, 269 (2003).

${ }^{3}$ K. Shariff and M. Wang, "A numerical experiment to determine whether surface shear-stress fluctuations are a true sound source," Phys. Fluids 17, 107105 (2005).

${ }^{4}$ A. Powell, "Aerodynamic noise and the plane boundary," J. Acoust. Soc. Am. 32, 982 (1960).

${ }^{5}$ M. J. Lighthill, "On sound generated aerodynamically: I. General theory," Proc. R. Soc. London, Ser. A 211, 564 (1952).

${ }^{6}$ G. P. Haddle and E. J. Skudrzyk, "The physics of flow noise," J. Acoust. Soc. Am. 46, 130 (1969).

${ }^{7}$ S. J. Barker, "Radiated noise from turbulent boundary layers in dilute polymer solutions," Phys. Fluids 16, 1387 (1973).

${ }^{8}$ E. M. Greshilov and M. A. Mironov, "Experimental evaluation of sound generated by turbulent flow in a hydrodynamic duct," Sov. Phys. Acoust. 29, 275 (1983).

${ }^{9}$ T. Colonius and S. K. Lele, "Computational aeroacoustics: progress on nonlinear problems of sound generation," Prog. Aerosp. Sci. 40, 345 (2004).

${ }^{10}$ Z. W. Hu, C. L. Morfey, and N. D. Sandham, "Wall pressure and shear stress spectra from direct numerical simulations of channel flow," AIAA J. 44, 1541 (2006)

${ }^{11}$ V. Arp, J. M. Persichetti, and G. B. Chen, "The Grüneisen parameter in fluids," J. Fluids Eng. 106, 193 (1984).

${ }^{12}$ P. M. Morse and K. U. Ingard, Theoretical Acoustics, 2nd ed. (Princeton University Press, Princeton, NJ, 1986).

${ }^{13}$ Z. W. Hu, C. L. Morfey, and N. D. Sandham, "Aeroacoustics of wallbounded turbulent flow," AIAA J. 40, 465 (2002). 